\title{
Demokratie und Zivilgesellschaft unter Druck. Anzeichen und Hintergründe einer weltweiten Krise
}

\begin{abstract}
Zusammenfassung
In dem Beitrag wird mit historisch-systematischem Interesse nach Erklärungen für den aktuellen Zustand und den damit verbundenen Handlungsoptionen wehrhafter Demokratien Ausschau gehalten. Dazu werden empirische Befunde und theoretische Überlegungen im weltweiten Kontext beschrieben. Mit Blick auf die Überlebensfähigkeit des Planeten wird die Notwendigkeit multilateralen politischen Handelns für Nachhaltigkeit in einer globalisierten Welt deutlich, die innovative Impulse braucht und den unverrückbaren universalen Menschenrechten verpflichtet bleibt. Jenseits einer Rückkehr zu ausgrenzenden Nationalstaaten erfolgt ein Plädoyer für streitbar-konstruktive Demokratie mit einem starken zivilgesellschaftlichen Fundament.
\end{abstract}

Schlüsselworte: Demokratie, Freiheit, Nachhaltigkeit, Zivilgesellschaft

\begin{abstract}
Based on a historic-systematic view, this article looks for explanations for the current state and the associated options for action of strong democracies. For this purpose, empirical findings and theoretical considerations are described in the global context. The need for multilateral political action for sustainability in a globalized world is described as necessary according to the planet's survivability. It becomes clear that all this needs innovative impetus and remains committed to immutable universal human rights. Beyond a return to exclusionary nation states, there is a plea for vibrant democracy with a strong civil society foundation.
\end{abstract}

Keywords: Democracy, freedom, sustainability, civil society

\section{Einleitung}

Es ist demokratischen Gesellschaften eigen, dass ihr Zustand von Teilen ihrer Öffentlichkeit als kritisch beschrieben wird. Die Demokratie steckt in einer Dauerkrise, könnte man jedenfalls im Rückblick auf die deutsche Demokratiegeschichte mei- nen. Krise ist eigentlich immer. So veröffentlichte auch in den turbulenten sechziger Jahren das Wochenmagazin „Die Zeit“ eine Reihe von Diskussionsbeiträgen zur Krise der Demokratie, deren Leitfragen Marion Gräfin Dönhoff zum Abschluss der Serie - und gewissermaßen zeitlos - zusammenfasste: „Ist die parlamentarische Demokratie noch fähig, mit den Konflikten der heutigen differenzierten Gesellschaft fertig zu werden? Ist sie noch in der Lage, ihre Aufgabe wahrzunehmen: politische Entscheidungen zu fällen und Kontrollen auszuüben?“ (Dönhoff, 1968).

Im Jahr 1969 gab der frisch gewählte deutsche Bundeskanzler Willy Brandt in seiner Regierungserklärung das Motto aus: „Wir wollen mehr Demokratie wagen!“ (Brandt, 1969). Dabei ging es freilich nicht um die Revision der parlamentarischen Demokratie und ihres Repräsentationsmodells, sondern um einen Demokratisierungsprozess, der über ordnungspolitische Fragen hinaus die Selbstbestimmungsprozesse in der Gesellschaft in den Blick nahm. Demokratisierung, wie sie damals nicht zuletzt die junge Generation vehement einklagte, zielte auf die Partizipation der Bürgerinnen und Bürger in allen Lebensbereichen, auf die Demokratisierung der Schulen und Universitäten, auf Mitbestimmung in den Betrieben, auf die Entrümpelung der Institutionen, auf die Liberalisierung der vorherrschenden Normen und Werte.

In diesem umfassenderen Verständnis ist Demokratisierung immer als ein unabgeschlossener Prozess, immer als unvollständig, als verbesserungsbedürftig zu begreifen. Demokratische Gesellschaften sind beständig im Wandel und müssen sich neuen Herausforderungen stellen. Das allein wäre noch kein Grund, eine Krise der Demokratie konstatieren zu wollen. Aber nach der sogenannten dritten Welle der Demokratisierung, seit Mitte der siebziger Jahre, die später auch viele Länder des ehemaligen Ostblocks erfasste und die Zahl der demokratisch regierten Länder sprunghaft in die Höhe schnellen ließ, ist wieder ein Schwund der Demokratien zu verzeichnen, der vielerorts zu neuen Krisenbefunden Anlass gibt. Demokratieforscher wie Wolfgang Merkel (2015) warnen zwar vor vor- 
eiligen alarmistischen Urteilen, konstatieren gleichwohl, dass die Demokratie vor ernstzunehmenden Herausforderungen steht. Allen Mahnungen zur nüchternen Betrachtung der Weltlage zum Trotz wird in diesem Beitrag von einem kritischen Zustand der demokratischen Entwicklung die Rede sein, ja von einer tiefgreifenden Krise der Demokratie. Denn es mehren sich die Anzeichen, dass Demokratie in ihren verschiedenen Dimensionen als Herrschafts-, Gesellschafts- wie als Lebensform gleichermaßen unter Druck gerät und dass demokratische und menschenrechtliche Errungenschaften der vergangenen Jahrzehnte erodieren. Gleichzeitig zeigt sich, dass diese Tendenzen eine weltweite Reichweite angenommen haben, zumindest aber Ausdruck eines globalen Trends sind, der auf Ursachen verweist, die jenseits der jeweils unterschiedlichen nationalstaatlichen Kontexte liegen. Auch viele der alten Demokratien des Westens sind diesem Druck ausgesetzt, der nicht zuletzt von einem autoritären Populismus beschleunigt wird, der sich unter der Maske einer demokratischen Bewegung verbirgt und längst zu einem weltweiten Phänomen geworden ist.

In einer zunehmenden Zahl von Ländern schwindet die Legitimation elementarer demokratischer Werte. Damit geraten auch die Grundlagen eines demokratischen Gemeinwesens ins Wanken. Aus der Summe sich häufender Einzelfälle demokratischer Erosionsprozesse kann inzwischen ein globaler Trend abgelesen werden, der auch große und historisch gewachsene demokratische Gesellschaften nicht unberührt lässt.

\section{Demokratie weltweit auf dem Rückzug}

Blicken wir zunächst auf einige empirische Befunde zum weltweiten Stand der demokratischen Entwicklung. Die wichtigsten internationalen Demokratie- und Freiheitsindizes sehen die Demokratie in jüngster Zeit auf dem Rückzug:

- Freedom House (2018) konstatiert in seinem Bericht „Freedom in the World 2018“, die Demokratie habe in 2017 ihre bislang schwerste Krise seit Jahrzehnten erfahren. Für das nunmehr zwölfte Jahr in Folge wird in diesem Report, der seit 1973 jährlich herausgegeben wird, festgestellt, dass sich die Einhaltung freiheitlicher und demokratischer Grundrechte fortlaufend verschlechtert habe. Grundrechte wie das Recht auf freie Wahlen, die Meinungsfreiheit, die Rechte von Minderheiten und die Rechtsstaatlichkeit seien weltweit unter Druck geraten. In 71 der 195 beobachteten Staaten würden politische Rechte der Bürgerinnen und Bürger weniger als zuvor geachtet, nur 35 Länder hätten Fortschritte zu verzeichnen. Nur 88 Nationen, das sind 45 Prozent der bewerteten Staaten, in denen 39 Prozent der Weltbevölkerung leben, werden von Freedom House noch als „frei“ eingestuft, demgegenüber 58 als „teils frei“ und 49 als „unfrei“ (Freedom House, 2018).

- Auch nach dem Transformationsindex BTI 2018 der Bertelsmann-Stiftung (2018) ist der Zustand von Demokratie und Marktwirtschaft in den hier untersuchten 129 Ländern auf den niedrigsten Stand seit 12 Jahren gefallen. In den vergangenen zwei Jahren habe sich nur in zwei Ländern die politische Situation verbessert (Burkina Faso und Sri Lanka), in 13 Staaten dagegen habe sie sich verschlechtert (darunter Polen, Ungarn, Türkei). Derzeit leben laut BTI 3,3 Milliarden Menschen in autokratischen Verhältnissen - mehr als jemals zuvor.
- Der Democracy-Index der Zeitschrift The Economist, der seit 2006 den Grad der Demokratie in 169 Ländern misst (The Economist Intelligence Unit, 2018), unterscheidet „vollständige Demokratien“, „unvollständige Demokratien“, „Hybridregime“ und „Autoritäre Regime“. Er stellt für 2017 fest, dass es nur noch 19 vollständige Demokratien gäbe. 2014 seien es noch 24 gewesen. Der Economist spricht von einer „Rezession der Demokratie“ (The Economist Intelligence Unit, 2018).

- Das Varieties of Democracy Project (Varieties of Democracy Institute, 2018) analysiert Demokratisierung nach einem multidimensionalen Ansatz mit inzwischen mehr als 450 differenzierten Indikatoren und Demokratieindizes und stellt in seiner jüngsten Bestandsaufnahme fest, dass sich die Anzahl der Demokratien weltweit seit 2012 in einem leichten Rückgang befindet, nachdem die Welt seit Mitte der 1970er Jahre eine stetige Ausbreitung von Demokratie erfahren habe. Nach wie vor bewege sich allerdings der Grad der Demokratisierung nahe an dem historischen Allzeithoch. Die Zahl der Menschen, die in autokratischen Systemen leben, wird hier mit 2,5 Milliarden angegeben. Autokratische Tendenzen zeichneten sich vor allem in bislang vorwiegend demokratischen Regionen ab. Nur 15 Prozent der Weltbevölkerung lebten in Staaten, in denen annährend Geschlechterparität beim Zugang zu politischer Macht hergestellt sei. Deutlich zugenommen habe der Ausschluss von politischer Teilhabe von Bevölkerungsgruppen mit einem schlechten sozioökonomischen Status.

- Der Civicus-Monitor (Civicus, 2018) hebt hervor, dass nach den Daten von 2018 nur vier Prozent der Weltbevölkerung, d. h. 282 Millionen Menschen, in Staaten leben, in denen sie ihre Grundrechte auf Meinungsäußerung, Versammlungs- und Vereinigungsfreiheit uneingeschränkt ausüben können. Demgegenüber sind immer mehr zivilgesellschaftliche Organisationen, unabhängige Journalistinnen und Journalisten und Menschenrechtsverteidiger staatlicher Repression, systematischer Kriminalisierung und gewaltsamen Angriffen ausgesetzt. Civicus, ein weltweites bürgerschaftliches Netzwerk, bemisst den zivilgesellschaftlichen Handlungsraum und konstatiert seit Jahren einen weltweiten Trend zum „shrinking space“ für das Handeln zivilgesellschaftlicher Akteure. Civicus kategorisiert die Freiheitsgrade einer Gesellschaft in fünf Kategorien: offen, beeinträchtigt, beschränkt, unterdrückt und geschlossen (Brot für die Welt \& Civicus, 2019, S. 10f.). Auf der Basis des Civicus-Monitors gibt Brot für die Welt seit Januar 2018 jährlich den deutschsprachigen Atlas der Zivilgesellschaft heraus. Die jüngste Ausgabe (Brot für die Welt \& Civicus, 2019) stellt fest, dass in nur 46 Staaten die zivilgesellschaftlichen Grundrechte voll respektiert und geschützt werden, ihr civic space wird somit als „offen" eingestuft. Dies ist u.a. in Deutschland, in Costa Rica und in Dänemark der Fall. In weiteren 40 Staaten ist es der Zivilgesellschaft ebenfalls grundsätzlich gestattet, ihre Versammlung-, Vereinigungs- und Meinungsfreiheit auszuüben, es kommt aber immer wieder zu Verletzungen dieser Rechte. Der civic space ist somit „beeinträchtigt“. Hierunter fallen z.B. Frankreich, USA und Österreich. 
„Beschränkt“, „,unterdrückt“ oder "geschlossen“ "sind laut Civicus die Handlungsräume der Zivilgesellschaft in 110 Staaten der Welt. Rund 2,7 Milliarden Menschen leben in 53 Staaten, z.B. Ungarn, Indien, oder Kenia, deren zivilgesellschaftlicher Handlungsraum „beschränkt“ ist. In 35 Staaten leben 1,4 Milliarden Menschen, die der Civicus-Monitor als ,unterdrückt“ einstuft. Dazu zählen Algerien, Bangladesch oder Venezuela. Auch in Europa zeigen sich solche Tendenzen. 54 Prozent der Europäer leben heute in Staaten, in denen der civic space „beeinträchtigt" oder „,beschränkt“ ist. Rund 3,4 Milliarden Menschen, die in „beschränkten“ oder „unterdrückten“ Ländern leben, riskieren, überwacht, drangsaliert oder inhaftiert zu werden. Vollständig "geschlossen“ ist der zivile civic space in 22 Staaten mit rund 2 Milliarden Menschen. In Ländern wie Ägypten, China, Nord-Korea oder Saudi-Arabien wird jede Kritik an der Regierung schwer bestraft, Medienfreiheit gibt es nicht, zivilgesellschaftliche Akteure werden misshandelt, inhaftiert oder auch getötet (Brot für die Welt \& Civicus, 2019).

Dergleichen Freiheits- und Demokratieindizes sind nicht unmittelbar miteinander vergleichbar, da sie unterschiedliche Indikatoren zugrundelegen bzw. diese verschieden gewichten. So unterscheiden sich auch die Länderratings vor allem in den mittleren Bereichen der Skala erheblich. Auch kann der hier besonders gewürdigte Civicus-Monitor dem eigenen Anspruch nach nicht als ein Demokratie-Index gewertet werden, da er sich vorrangig mit den Handlungsräumen der Zivilgesellschaft befasst. Die Einschränkung politisch-bürgerlicher Grundrechte und die damit verbundene Begrenzung zivilgesellschaftlicher Handlungsräume ist zwar nur ein Element des Demokratieabbaus, hat allerdings insofern zentrale Bedeutung, als eine lebendige Zivilgesellschaft das Fundament eines lebendigen demokratischen Gemeinwesens bildet.

Den genannten Freiheits- und Demokratieindizes ist der Befund eines insgesamt beunruhigenden weltweiten Trends gemeinsam, der angesichts zunehmender Einschränkung demokratischer Freiheitsrechte in vielen Ländern als eine weltweite Krise der Demokratie beschrieben wird. So konstatiert auch der Civicus Monitor eine „crisis of democracy" und ruft gar einen globalen Notstand aus, eine „global civic space emergency" (Civicus, 2018, o.S.).

\section{Defekte Demokratien}

Bei allen Bemühungen, den Entwicklungsstand einer Demokratie messen zu wollen, ist zu berücksichtigen, welches Demokratiekonzept jeweils zugrunde gelegt wird. Die Mehrzahl der etablierten Demokratieindizes ist recht minimalistisch. Sie stellen vor allem die Einlösung des Rechts auf freie und faire Wahlen in den Mittelpunkt und betrachten Demokratie in erster Linie in Analogie zu den frühen liberalen Modellen von Locke oder Tocqueville als ein Instrument, wie die Freiheitsrechte der Bürgerinnen und Bürger durch Übergriffe des Staates geschützt werden können. Die Autoren des von einer Forschergruppe um Marc Bühlmann und Wolfgang Merkel entwickelten „Demokratiebarometers" (Universität Zürich, 2018) halten es demgegenüber für erforderlich, die Qualität von Demokratie in einem weitaus umfassenderen Sinne zu be- werten und neben dem Schutz der Freiheitsrechte und den freien Wahlen auch die Mechanismen und das Ausmaß der politischen Beteiligung und Mitsprache der Bürgerinnen und Bürger sowie den Grad der sozialen Gerechtigkeit einzubeziehen - ein Anspruch, dem auch das Varieties of Democracy Project Rechnung trägt. Das Varieties of Democracy Projekt verwendet einen multidimensionalen Ansatz und disaggregierte Datensätze, die es z.B. auch erlauben, die Exklusion bestimmter gesellschaftlicher Gruppen von politischer Teilhabe oder das Ungleichgewicht der Geschlechter in der Ausübung politischer Positionen zu untersuchen.

Es liegt auf der Hand, dass die Durchführung von demokratischen Wahlen allein noch kein hinreichendes Charakteristikum eines demokratischen Systems sein kann, wenn andere wesentliche Elemente wie Rechtsstaatlichkeit, Gewaltenteilung und Teilhaberechte nicht gewährleistet sind. Hier wäre dann die Diagnose einer „defekten Demokratie“ (Merkel, Puhle, Croissant, Eicher \& Thiery, 2003) angebracht. Merkel et al. haben dies ausgehend vom Modell einer „eingebetteten Demokratie“ (Merkel, 2004) weiter entfaltet. Eine entwickelte, funktionierende Demokratie beruht demnach auf fünf ineinander „eingebettete“ Teilregime: „Fünf Teilregime definieren die eingebettete, rechtsstaatliche Demokratie: ein demokratisches Wahlregime (A), das Regime politischer Partizipationsrechte (B), das Teilregime bürgerlicher Freiheitsrechte (C), die institutionelle Sicherung der Gewaltenkontrolle (D) sowie die Garantie, dass die effektive Regierungsgewalt der demokratisch gewählten Repräsentanten de jure und de facto gesichert ist (E)." (Merkel, 2004, S. 7). Bei defekten Demokratien handelt es sich dann um „Herrschaftssysteme, die sich durch das Vorhandensein eines weitgehend funktionierenden demokratischen Wahlregimes zur Regelung des Herrschaftszugangs auszeichnen, aber durch Störungen in der Funktionslogik eines oder mehrerer der übrigen Teilregime die komplementären Stützen verlieren, die in einer funktionierenden Demokratie zur Sicherung von Freiheit, Gleichheit und Kontrolle unabdingbar sind“" (Merkel, 2003, S. 66).

Viele selbsternannte Demokratien sind meist keine (mehr). In vielen Staaten wird zwar noch der Schein demokratischer Formen aufrechterhalten, werden Wahlen abgehalten, die aber in erster Linie die Funktion haben, autoritäre Regime demokratisch abzusichern. Dergleichen Wahlautokratien sind eine Form hybrider Demokratien. In Bezug auf die internationale Entwicklungszusammenarbeit argumentiert Paul Collier (2009), dass die Fokussierung der Demokratieförderung auf Wahlen für viele arme Länder keine wirkliche Demokratisierung der Gesellschaften mit sich gebracht habe, sondern vielmehr Gewalt, ethnische Spaltung und Fragilität verschärfe. Viel zu wenig Augenmerk werde demgegenüber auf Rechtsstaatlichkeit und Gewaltenteilung als Konstituenten demokratischer Systeme gelegt.

Zieht man zudem in Betracht, dass selbst dort, wo die ordnungspolitischen Voraussetzungen demokratischer Regime noch funktionsfähig sind, der Raum des zivilgesellschaftlichen Engagements dennoch stark beschränkt sein kann und die Menschenrechte täglich verletzt werden, ist zu vermuten, dass die „Krise der Demokratie“ letztendlich noch sehr viel weitreichender ist, als minimalistische Demokratieindizes derzeit erkennen lassen. 
Es muss an dieser Stelle auch daran erinnert werden, dass die ersten beiden Demokratisierungswellen, die erste lange Welle von Mitte des 19. Jahrhunderts an und die zweite Welle nach dem Zweiten Weltkrieg, stets von autokratischen Gegenbewegungen gefolgt wurden, die viele der vorausgegangenen Errungenschaften $\mathrm{zu}$ zertrümmern vermochten, ohne freilich ein Wiedererstarken der demokratischen Kräfte verhindern zu können. Es ist nicht auszuschließen, dass auch die Stagnation, die eintrat, nachdem die dritte Welle ab Mitte der siebziger Jahre gegen Ende des 21. Jahrhunderts an Schwung verlor, nun in eine autokratische Gegenbewegung übergegangen ist, die wiederum nicht von langer Dauer sein wird und von neuen demokratischen Fortschritten eingeholt werden dürfte. Beunruhigend ist freilich die globale Dimension der sich abzeichnenden Veränderungsprozesse, die wiederum auf eine recht fundamentale Krise nationalstaatlicher Demokratie im Zeichen der Globalisierung verweisen.

\section{Auch der Multilateralismus verliert an Rückhalt}

In immer mehr Ländern schwindet die Anerkennung demokratischer Errungenschaften und menschenrechtlicher Prinzipien. Mit diesen Prozessen auf der nationalstaatlichen Ebene korrespondiert die Schwächung universeller Normen und Standards wie auch der multilateralen Institutionen, die zu deren Konsolidierung aufgebaut wurden. Das System einer regelbasierten internationalen Ordnung erodiert. Dadurch wird der Legitimationsschwund dieser Regeln auf nationaler Ebene wechselseitig verstärkt.

Nachdem die USA bereits im Sommer 2017 den Austritt aus dem Pariser Klimaabkommen angekündigt haben, sind sie in 2018 auch aus dem UN-Menschenrechtsrat ausgeschieden. Dem Ausstieg der USA aus dem Global Compact on Migration der Vereinten Nationen haben sich in 2018 eine ganze Reihe von Staaten, wie Ungarn, Australien, Polen und Österreich angeschlossen. Die Zahlungen für das Flüchtlingshilfswerk der UN für Palästina UNRWA werden von den USA laut Ankündigung von Ende August 2018 vollständig eingestellt. Die in den Vorjahren stets im Konsens verabschiedete Ministererklärung des High Level Political Forum der Vereinten Nationen zu den Zielen für eine nachhaltige Entwicklung verweigerten sich im Juli 2018 erstmals zwei Staaten: die USA und Israel. Das ist auch als Signal zu verstehen, dass beide Staaten sich der weiteren Beteiligung an der gemeinsamen Umsetzung der SDGs entziehen.

Ein regelbasiertes globales Handelssystem befindet sich im Zeichen zunehmender Handelskriege und bilateraler Abkommen im freien Fall. Der Multilateralismus steckt in zahlreichen Politikfeldern in der Krise. Japan tritt nach drei Jahrzehnten aus dem völkerrechtlichen Vertrag der Internationalen Walfangkommission aus. Zahlreiche afrikanische Länder kehren dem Internationalen Strafgerichtshof (dem die USA, China und Russland ohnehin nicht angehören) den Rücken. Auch die Philippinen erwägen einen Austritt. Die Union Südamerikanischer Nationen (UNASUR) zeigt Auflösungserscheinungen. Urteile des Europäischen Gerichtshofs für Menschenrechte werden von einer Reihe europäischer Staaten ignoriert. Auch die Interamerikanische Menschenrechtskommission verliert an Anerkennung und erhält keine ausreichende Finanzierung mehr. Weltweit wächst die Tendenz, Entscheidungskompetenzen zu re-nationalisieren. Das trifft nicht zuletzt auch auf die Europäische Union zu. Die EU-Institutionen verlieren an Einfluss und Handlungsmacht. Gleichzeitig gibt es aber auch Bemühungen und Tendenzen, weitere Politikbereiche in der EU stärker zu vergemeinschaften wie z. B. die Entwicklungsund die Außenpolitik. Der Ausgang der Europawahl in diesem Jahr und die Folgen des Brexits könnten freilich solche Bemühungen beenden und eine weitere Schwächung der EU und gar ihr Auseinanderbrechen mit sich bringen.

Aber für die EU selbst wie auch für die multilateralen Strukturen auf globaler Ebene gilt, dass der Krise des Multilateralismus nicht einfach mit einem Festhalten oder mit der Restauration der alten Strukturen und Institutionen begegnet werden kann. Die Reform der EU-Institutionen wie auch die der Vereinten Nationen, der WTO etc. bleibt auf der Tagesordnung, gerade wenn es darum geht, regionale und globale Governance im Sinne einer gerechten und nachhaltigen Entwicklung zu stärken.

Paradoxerweise bringt es gerade die fortschreitende Schwächung der ohnehin nur wenig schlagkräftig ausgeprägten Global Governance-Strukturen mit sich, dass die politische Steuerbarkeit grenzüberschreitender Prozesse weiter abnimmt. Aber gerade dieser allenthalben beobachtete Kontrollverlust der Politik gegenüber der Globalisierung scheint ein wichtiger Faktor für die zur Frage stehende Krise der Demokratie zu sein.

\section{Grenzen und Herausforderungen nationalstaatlicher Demokratie im Zeitalter der Globalisierung}

Die Globalisierung, im Sinne der Ausdehnung, Verdichtung und Beschleunigung grenzüberschreitender sozialer und ökonomischer Interaktionen, hatte zunächst, nach dem Fall der Mauer, die Ausbreitung eines liberalen Gesellschafts- und Wirtschaftsmodells westlicher Prägung erheblich vorangebracht. Angesichts der wachsenden Bedeutung transnationaler Prozesse, insbesondere der Macht eines grenzüberschreitend organisierten Finanz- und Wirtschaftssystems, wird nun allerdings immer deutlicher erfahren, dass die Gestaltungsfähigkeit der Nationalstaaten auch in den Fragen schwindet, die ihre eigenen Angelegenheiten betreffen. Dies wird häufig als Souveränitäts- und Kontrollverlust begriffen und mündet beispielsweise in Teilen der Bevölkerung in der massiven Kritik am vermeintlichen Versagen der politischen Elite. Die schwindende politische Partizipation, insbesondere angesichts des Einflusses der multinationalen Unternehmen ist für Colin Crouch (2004) ein Indiz dafür, dass die globalisierte Welt inzwischen im Stadium von "Postdemokratien“ angekommen sei. Wahlen degenerierten in der Postdemokratie zum reinen Spektakel.

Eine wachsende Zahl von Menschen sympathisiert als Reaktion auf die Denationalisierung weiter Bereiche des gesellschaftlichen Lebens mit nationalistischen Gegenstrategien. Dergleichen Positionen gehen mit Plädoyers für eine Stärkung der nationalen Identität und des Nationalstaats, für einen Abbau grenzüberschreitender Beziehungen und der internationalen Kooperation, aber auch mit Ausgrenzung gegenüber Migrantinnen und Migranten, mit Fremdenfeindlichkeit und rassistischen Ressentiments einher. 
Denkbar ist freilich auch eine kosmopolitische Option als Antwort auf die Grenzen nationalstaatlicher Demokratie in einer globalisierten Welt. Diese Option geht davon aus, dass das Primat der Politik über eine grenzenlos operierende Ökonomie nur durch Stärkung von Global Governance wiedererrungen werden kann. Kosmopolitische Optionen sind derzeit freilich weniger populär. Dabei lässt sich durchaus zeigen, dass der Souveränitätsverzicht infolge internationaler Kooperation mit Kooperationsgewinnen einhergeht, der auf längere Sicht in einen Zugewinn an politischer Souveränität aller daran Beteiligten mündet (Kappes \& Seitz, 2015): Politische Souveränität ist in einer globalisierten Welt und angesichts grenzüberschreitender Herausforderungen letztlich nur im Rahmen grenzüberschreitender Kooperation zu gewährleisten, und gewiss nicht durch Abschottung und eine Betonung des Leitprinzips nationaler Eigeninteressen.

Die transnationale Zivilgesellschaft ist ein wichtiger Motor der internationalen Kooperation und der Suche nach transnationalen Lösungen. Das zeigt sich mehr und mehr auch in der Rolle, die sie in zentralen multilateralen Politikprozessen, z.B. bei den Klimaverhandlungen oder im Menschenrechtsrat einnimmt. Für die Wahrnehmung ihrer Watchdogfunktion ist der Zivilgesellschaft die Bezugnahme auf weltweit gültige Maßstäbe, Umwelt- und Menschenrechtstandards ganz besonders bedeutsam. Häufig rücken dabei auch Konflikte zwischen zivilgesellschaftlichen Positionen und den Interessen der Privatwirtschaft ins Zentrum. Zivilgesellschaftliche Organisationen setzen sich zur Wehr, wenn Investoren und Unternehmen gegen nationale Gesetze oder gegen Umwelt- und Sozialstandards verstoßen, mit der Folge, dass sie kriminalisiert oder diffamiert werden (Forum Menschenrechte et al., 2017). Oftmals ist der Widerstand gegen die Sicherung wirtschaftlicher Partikularinteressen ein zentraler Grund für die Repression gegenüber der Zivilgesellschaft. Daher sind es nicht nur autokratische und rechte nationalistische Regime, die zivilgesellschaftliche Handlungsspielräume einschränken, sondern zunehmend auch neoliberal orientierte Regierungen, die die Interessen der Wirtschaft höher bewerten als das Gemeinwohl.

Neben den Spannungen, die sich im Zuge der Globalisierung aus dem Wachstum der ökonomischen Macht und der Schwächung der nationalstaatlichen Steuerungskompetenz ergeben, werden von der Demokratieforschung (u.a. Merkel, 2015) im Blick auf die OECD-Welt für Politikverdruss, Wahlmüdigkeit und damit einhergehende Aushöhlung der Demokratie zahlreiche weitere Faktoren ins Feld geführt, darunter

- die Abkoppelung der gesellschaftlichen Eliten,

- die Krise der Volksparteien,

- die wachsende sozioökonomische Ungleichheit,

- die Begrenzung von Freiheitsrechten unter den Vorzeichen der Terrorismusbekämpfung und der Bedrohungen der öffentlichen Sicherheit und

- der empfundene Verlust an „Zugehörigkeit“ zu einer klar definierten umgrenzbaren Gemeinschaft

Große Aufmerksamkeit gilt in jüngster Zeit zudem der Rolle der Digitalisierung und der modernen Kommunikationsmedien. Sie wird im Blick auf die Auswirkungen auf die Demokratie sehr ambivalent beurteilt: positiv sind die gewaltig gewach- senen Möglichkeiten der Informationsbeschaffung und der Geschwindigkeit und Reichweite der Kommunikation und Meinungsäußerung zu nennen, negativ die Tendenz, dass sich bestimmte Bevölkerungskreise gar nicht mehr mit ihnen unangenehmen Positionen und Nachrichten auseinandersetzen, sondern in einer kommunikativen „Filterblase“ verharren, wodurch die Segmentierung der demokratischen Öffentlichkeit weiter voranschreitet. Als große Herausforderungen für die Zukunft der Demokratie sind auch die zunehmende Orientierungslosigkeit angesichts der Inflationierung und Beliebigkeit der Kommunikationsofferten, die Zunahme der Manipulation von Öffentlichkeit durch gezielte Fehlinformationen und Fake News wie auch durch die exzessive Nutzung personenbezogener Daten zu nennen (Welzer, 2008).

\section{Zivilgesellschaft als Fundament eines demokratischen Gemeinwesens}

Die Bedeutung der Zivilgesellschaft für eine nachhaltige Entwicklung wurde von wichtigen internationalen Prozessen anerkannt. Bereits in der Agenda 21, die bei der Weltkonferenz für Umwelt und Entwicklung 1992 verabschiedet wurde, wurden der Einbeziehung der Zivilgesellschaft und den Nichtregierungsorganisationen eine Schlüsselrolle für die Umsetzung des hier beschlossenen Leitbildes einer nachhaltigen Entwicklung beigemessen. Gut ein Drittel der Artikel dieses Dokuments befasst sich mit der Rolle der Öffentlichkeit und der Zivilgesellschaft. Damit trug der UN-Gipfel der Einsicht Rechnung, dass staatliche Instrumente alleine nicht ausreichen, um das damals formulierte ambitionierte Aktionsprogramm in allen Bereichen der Gesellschaft umzusetzen. Die Globale Partnerschaft (Global Partnership on Effective Development Cooperation) bekräftigte in ihrer Nairobi-Erklärung 2016 die Rolle der Zivilgesellschaft als eigenständiger Entwicklungsakteur und erwähnte explizit die Wichtigkeit des unabhängigen und kritischen Engagements. In der Erklärung wurden außerdem „Shrinking Civic Space“ (Global Partnership, 2016) zusammen mit Aspekten wie Klimawandel und Wirtschaftskrisen als Entwicklungsrisiken identifiziert. Der Generalsekretär der Vereinten Nationen betont, dass die Zivilgesellschaft Katalysator für sozialen Fortschritt und wirtschaftliches Wachstum sei. Sie spiele eine wesentliche Rolle, um Regierungen zur Rechenschaft zu ziehen und die verschiedenen Interessen einer Gesellschaft, vor allem deren benachteiligte Teile, zu repräsentieren. Und für die Agenda 2030 und ihre Ziele für eine nachhaltige Entwicklung gilt erst recht: Ohne eine Teilhabe der breiten Öffentlichkeit, ohne Sachkompetenz und Engagement der Bürgerinnen und Bürger und ohne die Mitwirkung der zivilgesellschaftlichen Akteurinnen und Akteure kann die Wende in Richtung Nachhaltigkeit und Gerechtigkeit nicht gelingen.

Zivilgesellschaft meint dabei sehr viel mehr als nur das Netzwerk der sogenannten Nichtregierungsorganisationen. Zunächst einmal ist unter Zivilgesellschaft jene Sphäre des öffentlichen kollektiven Handelns zu verstehen, die sich zwischen Wirtschaft und Staat befindet und die durch ein hohes $\mathrm{Maß}$ an Selbstorganisation geprägt ist. Neben dieser Charakterisierung von Zivilgesellschaft als eines bestimmten gesellschaftlichen Sektors kann sie darüber hinaus aber auch durch eine spezifische Differenz ihrer Handlungslogik unterschieden 
werden: Zivilgesellschaftliches Handeln folgt weder der Logik des Marktes noch der des politischen Machterhalts. Ihm geht es vielmehr darum, die Gemeinwohlorientierung der Gesellschaft zu stärken und die Voraussetzungen dafür zu schaffen, dass kollektive öffentliche Güter, die weder Markt oder Staat bereitstellen können, geschaffen und erhalten werden. Zivilgesellschaftliches Handeln ist auf die Gestaltung politischer Lebenszusammenhänge und nicht auf private Interessen ausgerichtet. Innerhalb des zivilgesellschaftlichen Raumes agieren Initiativen und soziale Bewegungen, Nichtregierungsorganisationen und Netzwerke, die weder marktwirtschaftlich-profitorientiert noch staatlich organisiert sind. Häufig wird daher auch die Summe der Akteurinnen und Akteure, die im Raum der Zivilgesellschaft tätig sind, mit der Zivilgesellschaft selbst identifiziert, was allerdings verkürzt erscheint, wenn ihre normative Fundierung nicht auch in den Blick genommen wird.

Maina Kiai, der UN-Sonderberichterstatter für das Recht auf Versammlungs- und Vereinigungsfreiheit (Mai 2011 bis April 2017) hat sich in seinem letzten Bericht an den Menschenrechtsrat der Vereinten Nationen dieses dreidimensionale Verständnis von Zivilgesellschaft in der Trias „forms, norms, spaces“ in Anlehnung an Michael Edwards, ausdrücklich zu eigen gemacht: „The Special Rapporteur has adopted an understanding of civil society as embodying, forms' (diverse associational relationships), embracing, norms' (values that shape a "good society", such as freedom, democracy, tolerance and cooperation), and engaging in ,spaces' (the public sphere where discussions and disputes can freely take place with a view to achieving consensus on what is good for society)." (UN, 2017, S. 4).

Für die normativen Grundlagen zivilgesellschaftlichen Handelns ist entscheidend, dass sie sich an der Würde eines/ einer jeden einzelnen Menschen orientiert. Die Anerkennung der Würde eines jeden Menschen, die der eigenen Person ausdrücklich eingeschlossen, ist conditio sine qua non einer demokratischen Gesellschaft. Wo Hass und Ausgrenzung, Gewalt gegen Andersdenkende, Diskriminierung und Marginalisierung regieren und sich eine „uncivil society“ breit macht, ist es um die Zukunft der Demokratie schlecht bestellt.

Wenn die Meinungs- und Versammlungsfreiheit massiv beschnitten wird, wenn Kritikerinnen und Kritiker zum Verstummen gebracht werden und politische Einmischung unter Strafe steht, so hat dies negative Auswirkungen auf die soziale, kulturelle und ökonomische Entwicklung. Nicht von ungefähr korreliert, nach den Ergebnissen des Civicus-Monitors, der Grad der menschlichen Entwicklung in einem Land (gemessen nach dem Human Development Index) eng mit dem Entwicklungsstand und den Freiheitsgraden der jeweiligen Zivilgesellschaft. Die Zivilgesellschaft ist, wenn sie sich frei entfalten kann, ein wichtiger Garant, Motor und ein wichtiges Korrektiv für eine funktionierende Demokratie, sie verhilft den Schwachen zur Artikulation und Berücksichtigung ihrer Interessen, sie ist Impulsgeberin und Mediatorin, sie gestaltet das bürgerschaftliche Engagement vor Ort, sie ist Akteurin gesellschaftlicher Transformation hin zu einer demokratischen und nachhaltigen Entwicklung, und sie schafft als transnational vernetzte Zivilgesellschaft die Grundlagen für eine am globalen Gemeinwohl orientierte internationale Politik.

\section{Fazit}

Der kursorische Blick auf die aktuellen Gefährdungen, denen die Demokratie heute ausgesetzt ist, demonstriert zunächst einmal: Demokratie ist zerbrechlich. Wir dürfen nicht dem Irrtum verfallen, dass die Existenz bestimmter staatlicher Institutionen, die Etablierung von Gewaltenteilung und Rechtsstaatlichkeit oder die regelmäßige Durchführung von Parlamentswahlen bereits hinreichen, um ein demokratisches Gemeinwesen nachhaltig zu garantieren. Demokratie ist nur als anhaltende Demokratisierung möglich. Demokratie ist kein einmal erreichter Zustand, sondern eine andauernde Herausforderung.

Funktionierende demokratische Institutionen sind eine notwendige, aber nicht hinreichende Voraussetzung einer demokratischen Gesellschaft. Karl Popper stellte in einem 1958 gehaltenen Vortrag die wichtige Frage: „Wie können wir unsere politischen Einrichtungen so aufbauen, dass auch unfähige und unredliche Machthaber keinen großen Schaden anrichten können?" (Popper, 1984). Freilich ist zu befürchten, dass der Schaden durchaus groß sein kann, wenn man sich allzu sehr auf die Tragfähigkeit der Institutionen verlässt. Allenthalben ist derzeit spürbar, wie der Aufstieg der Rechtspopulisten, ihr Einzug in föderale und nationale Parlamente wie auch in die Regierungen das gesellschaftliche Klima tiefgreifend verändern. Eine demokratische Öffentlichkeit wird systematisch zerrüttet. Die Erwartungen vieler Demokratinnen und Demokraten, dass die Ergebnisse kommender Wahlgänge dem Spuk wieder ein Ende machen werden, sind trügerisch. Die demokratischen Kräfte hüllen sich vielfach in Schweigen. Sie haben die Hoffnung, dass das demokratische System stark genug sei, diese Angriffe auf die Demokratie abzuwehren. Doch dieses Vertrauen ist trügerisch. Demokratie kann nicht einfach überwintern. Demokratisierung ist wie Fahrradfahren: Wenn wir aufhören, in die Pedale zu treten, dann fallen wir um.

Demokratie ist eine Lebensform und eine permanente Bildungsaufgabe. Demokratie ist ein unabgeschlossener Prozess, in dem der Streit über das, was eine demokratische Gesellschaft sein soll, immer auch Teil der öffentlichen Auseinandersetzung sein muss. Der Weg zu einer demokratischen nachhaltigen Entwicklung beginnt damit, dass engagierte Bürgerinnen und Bürger überall in der Welt die Möglichkeit haben, an der Bestimmung dessen, worin eine nachhaltige Entwicklung ihrer Gesellschaft besteht, mitwirken und mitentscheiden zu können. Im Sinne eines partizipatorischen und umfassenden Demokratieverständnisses bedarf es heute vor allem der Verteidigung und Öffnung des zivilgesellschaftlichen Handlungsraums und der Erweiterung der institutionellen Beteiligungsformate der zivilgesellschaftlichen Akteure, unabhängig vom Grad ihrer Organisiertheit, von Bürgerinitiativen über soziale Bewegungen bis hin zu den professionellen Nichtregierungsorganisationen, um eine Demokratisierung der bestehenden Demokratieformate voranzubringen.

Das Aufbegehren für mehr Demokratie war und ist Ausdruck einer globalen Bewegung. Diese Bewegung muss auch ihr Demokratieverständnis globalisieren und sich der Entfaltung der demokratischen Potenziale auf allen gesellschaftlichen Ebenen, von der lokalen bis hin zur globalen Ebene annehmen. Die Zukunft der Demokratie kann nur noch kosmopolitisch gedacht werden. 


\section{Literatur}

Bertelsmann Stiftung (2018). Transformation Index BTI. Zugriff am 28.12.2018 https://www.bti-project.org/en/home/

Brandt, W. (1969). Regierungserklärung vor dem Deutschen Bundestag in Bonn am 28. Oktober 1969, hrsg. von der Bundeskanzler-Willy-Brandt-Stiftung, Zugriff am 28.12.2018, https://www.willy-brandt.de/fileadmin/brandt/Downloads/Regie rungserklaerung_Willy_Brandt_1969.pdf

Brot für die Welt \& Civicus (2019, i.E.). Atlas der Zivilgesellschaft, Report zur weltweiten Lage. Berlin: Brot für die Welt.

Civicus (2018). State of Civil Society Report 2018. Zugriff am 28.12.2018 https:// monitor.civicus.org/SOCS2018/

Collier, P. (2009). Gefährliche Wahl. Wie Demokratisierung in den ärmsten Ländern der Erde gelingen kann. Berlin: Siedler.

Crouch, C. (2004). Post-Democracy. Oxford: Blackwell Publishers.

Dönhoff, M. Gräfin (1968). Krise der Demokratie? Resümee einer Diskussion. Die Zeit Nr. 05/1968, Zugriff am 28.12.2018, https://www.zeit.de/1968/05/krise-derdemokratie

Forum Menschenrechte, Forum Umwelt und Entwicklung, VENRO - Verband Entwicklungspolitik und Humanitäre Hilfe, deutscher Nichtregierungsorganisationen e.V., Konsortium ZFD, Amnesty International, Brot für die Welt - Evangelischer Entwicklungsdienst Evangelisches Werk für Diakonie und Entwicklung e.V., Medica Mondiale, Medico International, Misereor, Oxfam, Reporter ohne Grenzen, Welthungerhilfe (2017). Zivilgesellschaftliches Engagement weltweit in Gefahr. Für gerechte Entwicklung, Umweltschutz, Demokratie, Menschenrechte und Frieden. Berlin, Zugriff am 28.12.2018 https://venro.org/fileadmin/user_upload/Dateien/Daten/Publikationen/Diskussionspapiere/Zivilgesellschaftliches_Engagement_weltweit_in_Gefahr_-_Forderungspapier_final_DIGITAL.pdf.

Freedom House (2018), Freedom in the World 2018, Zugriff am 28.12.2018, https://freedomhouse.org/report/freedom-world/freedom-world-2018

Global Partnership for Effective Development Cooperation (2016). Nairobi Outcome Document, Zugriff am 28.12.2018, http://effectivecooperation.org/wpcontent/uploads/2016/12/OutcomeDocumentEnglish.pdf
Kappes, B. \& Seitz, K. (2015) (Hrsg.). Nachhaltige Entwicklung braucht Global Governance, Weltinnenpolitik für das 21. Jahrhundert. München: Oekom.

Merkel, W. (2004). Die „eingebettete“ Demokratie. Ein analytisches Konzept. WZB-Mitteilungen, 106, 7-10.

Merkel, W. (2015) (Hrsg.). Demokratie und Krise, Zum schwierigen Verhältnis von Theorie und Empirie. Wiesbaden: Springer.

Merkel, W., Puhle, H.J., Croissant, A., Eicher, C. \& Thiery, P. (2003). Defekte Demokratie. Bd. 1: Theorie. Opladen: Leske + Budrich. doi.org/10.1007/978-3-66309573-6

Popper, K. R. (1984). Woran glaubt der Westen? In K. R. Popper (Hrsg.), Auf der Suche nach einer besseren Welt (S. 231-253). München: Piper.

The Economist Intelligence Unit (2018). Democracy Index 2017, Free speech under attack. London. Zugriff am 28.12.2018, http://www.eiu.com/topic/democracyindex.

UN (2017). Report of the Special Rapporteur on the rights to freedom of peaceful assembly and of association, Human Rights Council. Thirty- fifth session 6-23 June 2017, A /HRC/35/28.

Universität Zürich (2018). Democraybarometer. Zugriff am 28.12.2019, http:// www.democracybarometer.org/index.html

Varietes of Democracy Institute (2018). Democracy for All? V-Dem Annual Democracy Report 2018, University of Gothenburg, Zugriff am 28.12.2018 https:// www.v-dem.net/en/news-publications/democracy-reports/

Welzer, H. (2008). Die smarte Diktatur. Der Angriff auf unsere Freiheit. Frankfurt/M.: S. Fischer.

\section{Dr. phil. habil. Klaus Seitz}

ist Leiter der Abteilung Politik von Brot für die Welt, Berlin; langjährige Tätigkeit als Redakteur von epd-Entwicklungspolitik, Privatdozent für internationale Bildungsforschung an der Universität Hannover, Geschäftsführer des Ausschusses für entwicklungsbezogene Bildung und Publizistik der EKD und stellvertretender VENRO-Vorsitzender (bis 2017); zahlreiche Veröffentlichungen zur Entwicklungspolitik, Entwicklungspädagogik, Globalem Lernen und weltbürgerlicher Erziehung.

\section{UNSERE BUCHEMPFEHLUNG}

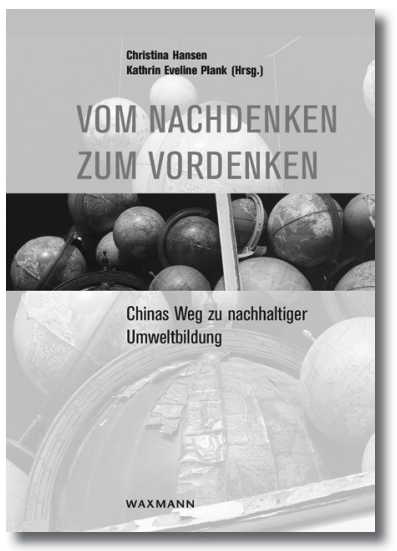

2019, 142 Seiten, br., 29,90€, ISBN 978-3-8309-3889-7

E-Book: $26,99 €$, ISBN 978-3-8309-8889-2
Christina Hansen, Kathrin Eveline Plank (Hrsg.)

\section{Vom Nachdenken zum Vordenken Chinas Weg zu nachhaltiger Umweltbildung}

Bildung ist ein Schlüsselfaktor für nachhaltige Entwicklung, darüber ist sich die Weltgemeinschaft einig.

Damit dies an Schulen gelingt, müssen bereits in der Lehrerbildung jene Voraussetzungen geschaffen werden, mit denen angehende Lehrkräfte Kompetenzen erwerben, die sie in die Lage versetzen, Fragen nachhaltiger Entwicklung unter globaler Perspektive zu reflektieren und didaktisch aufzubereiten.

Dies stellt aber eine große Herausforderung für die Lehrerbildung dar, denn es bedarf dafür mehrperspektivischer und fachübergreifender Konzepte, die auch anschlussfähig sind an unterschiedliche nationale Studienstrukturen. Schließlich gilt es, diese Konzepte für die Schule transferfähig zu machen.

Wie eine solche Umsetzung auf allen Ebenen der Lehrerbildung zu realisieren ist, zeigt die vorliegende deutsch-chinesische Forschungsarbeit, in der ein solches Konzept entwickelt und anschließend mit 19 Schulen aus drei Provinzen in die nationale Lehrerbildung in China implementiert wurde. 